\title{
The in vitro Effects of Tryptamine, Harmine, and Harmaline on Leishmania tarentolae and the Possible Implications for Leishmaniasis
}

\author{
Jessica Wickline, Alexis Broedlow, Benjamin M Dorsey, Kirk Eichenberg and Marjorie A Jones* \\ Department of Chemistry, Illinois State University, Normal, IL 61790-4160, USA
}

\begin{abstract}
Leishmaniasis is a disease caused by Leishmania parasitic protozoans affecting people in both the Eastern and Western Hemispheres. The secreted acid phosphatase enzymes (SAPs) are reported to play a critical role in infection by Leishmania. Thus, these enzymes are potential targets for Leishmania therapy. Tryptamines have various physiological effects and thus serve different purposes socially. Tryptamines are used in ritualistic ceremonies in countries where Leishmania cases are reported. In this work, tryptamine and two other indole derivatives, harmine and harmaline, were investigated. Harmine and harmaline were selected because of their presence in the biological materials used in some South American ritualistic ceremonies. We investigated the effects on axenic Leishmania tarentolae cell shape, motility, clumping, and viability as well as on the activity of secreted acid phosphatase (SAP) from L. tarentolae. An overall decrease in cell viability over a seven-day period and a small recovery in cell viability, only at lower concentrations of test compounds, were observed. These compounds were, in general, activators of $L$. tarentolae SAP activity. This is the first report of effects of these compounds on Leishmania secreted acid phosphatase activity in vitro. We speculate that those with Leishmania infections may be worsening their condition with the exposure to these compounds.
\end{abstract}

\section{Introduction}

\section{Epidemiology of Leishmaniasis and Treatment Options}

Leishmaniasis is a disease caused by the parasitic protozoan Leishmania [1]. This disease affects people in more than 90 countries in the Eastern and Western hemispheres with cases reported on every continent except Australia and Antarctica. The exact number of leishmaniasis cases is not known but is reported to be between 1.5 and 2.0 million new cases per year [2] and over 150 million people affected worldwide [3]. Leishmaniasis is caused by 20 different species of Leishmania and is spread by the bite of some 30 different species of phlebotomine sandflies. The species of Leishmania, or sandfly, responsible for host infection are different for different hosts. The specific species of Leishmania or sandfly are reported as an associative relationship defined on the different hosts that are infected [2]. Leishmaniasis is presented clinically in three different forms: cutaneous, visceral, or mucocutaneous [4]. Cutaneous leishmaniasis presents as skin lesions that develop on the time scale of a week to a month. Patients with visceral leishmaniasis present with symptoms of fever, weight loss, swelling of the liver and spleen, low red blood cell count, and/or low white blood cell counts. These symptoms typically develop on the time scale of months to years. Mucocutaneous leishmaniasis is less common. It is typically seen as the consequence of a cutaneous leishmaniasis infection where the parasites have spread from the skin to the mucus membranes in the mouth, throat, or most commonly, the nose [4].

The treatment of leishmaniasis is not trivial. Treatment depends on both the parasite and the host. Some treatments are only effective for certain species of Leishmania [5]. Furthermore, data collected from clinical trials prove to be difficult to generalize across regions or the specific species of Leishmania parasite. Children, the elderly, and pregnant or lactating women may need different medication or dosage [5]. Walton and Valverde (1979) also reported that there were racial differences in resistance to leishmanial infections in South American native tribes relative to persons of African ancestry [6]. Regardless, there are three main therapy types, depending on the clinical presentation and parasite species, including: systemic therapy (parenteral), systemic therapy (oral), or local therapy. Systemic parental therapies include sodium and liposomal drug formulations of pentavalent antimony $\left(\mathrm{Sb}^{+5}\right)$ compounds. Systemic oral therapy includes ketoconazole, itraconazole, and fluconazole. The local therapies include liposomal amphotericin B, amphotericin B deoxycholate, pentavalent antimony $\left(\mathrm{Sb}^{+5}\right)$, or miltefosine [5]. These treatments are both expensive and result in harsh side effects with reports of drug resistance becoming a serious problem [3]. The total direct cost of treatment for an adult with visceral leishmaniasis is in the range of several hundred to tens of thousands of dollars and depends on both the drug used and the duration of the treatment [7]. However, use of local plant materials, in various types of ceremonies, in areas where leishmaniasis is endemic has not yet been well studied. This should be of interest since various plant materials may have positive or negative effects on the severity of the parasitic infections.

${ }^{*}$ Correspondence to: Marjorie A Jones, Department of Chemistry, Illinois State University, Normal, IL 61790-4160, USA, E-mail: majone3@ilstu.edu

Key words: tryptamine, harmine, harmaline, Leishmania, secreted acid phosphatase

Received: September 16, 2019; Accepted: September 25, 2019; Published: September 28, 2019 


\section{Leishmania Infection Cycle and the Role of Secreted Acid Phosphatases}

Leishmaniasis is transmitted from the bite of the infected female phlebotomine sandfly vector. There are eight steps and two general stages of the leishmaniasis infection cycle (Figure 1). This cycle is characterized as anthroponotic; thus, humans are needed to continue the human-sandfly-human cycle of transmission [8]. During the infection cycle, the Leishmania parasites exist in two major forms at different times. These two forms are the promastigote form and the amastigote form [9]. Leishmania secrete acid phosphatases, and these secreted acid phosphatases (SAP) are reported to have roles in the Leishmania infection cycle [10]. There are two Leishmania secreted acid phosphatase genes reported, LmSAP1 and LmSAP2 [11]. These genes are reported to be similar in amino acid sequence, with the largest differences being observed in the size of the serine/threonine rich domain that becomes heavily glycosylated during post translational modifications [12]. The SAP enzymes are critical virulence factors and are reported to play roles in the in-host transformation of the Leishmania promastigote form to the amastigote form [11]. These enzymes are also thought to play a significant role in the infection cycle due to their not yet welldefined interaction with host macrophages $[10,13]$. Furthermore, when the gene loci for these SAPs were deleted in the L. Mexicana species, the virulence of the resulting parasites was abolished [11]. Thus, these enzymes and their response to potential inhibitors or activators are of interest as potential targets for leishmaniasis therapy especially at early stages of infection by the promastigotes.

\section{The Chemistry, Biochemistry, and Importance of Tryptamines}

Tryptamines are a class of monamine indole alkaloids with biological activity [14]. Pharmacologically, tryptamine compounds are known to be serotonergic agonists of the 5-hydroxytryptamine receptor (5HTR) having profound biological effects on human thought and mood [15]. Because of this, biological materials containing tryptamines are often used in ritualistic ceremonies in South America, a geographical location where cases of leishmaniasis are reported [15]. Ayahuasca is a psychotropic beverage rich in psychedelic indole derivates long used in the amazon basin regions [16,17]. Tryptamine itself also acts indirectly as a vasoconstrictor via the discharge of noradrenaline from the sympathetic nervous system [18]. The tryptamine analogue 5-carboxamidotryptamine is a powerful vasodilator in vivo through its action on smooth muscle [19]. Furthermore, there are tryptamine derivatives with known anti-microbial or anti-fungal activity. These compounds are tryptamine-based sulfonamide-compounds, such as $\mathrm{N}-(2-(1 \mathrm{H}-$ Indole-3-yl) ethyl)-4-fluorobenzenesulfonamide $[20,21]$. Other molecules in the tryptamine family have effects on or serve as diurnal rhythm modulators, migraine treatments, anti-depressants, stimulants, psychedelics, monoamine oxidase inhibitors, nausea suppressive agents, anti-oxidants, or cytoprotective agents [21]. Thus, there are many direct and indirect physiological effects caused by exposure to the tryptamine class of indole derivatives that have been reported. In this work, we focus on investigating the effects on axenic Leishmania of tryptamine (Figure 2a), and the two tryptamine indole pyridyl-derivatives: harmine (Figure $2 \mathrm{~b}$ ) and harmaline (Figure $2 \mathrm{c}$ ). We selected tryptamine, harmine, and harmaline due to their presence in biological materials used in ritualistic ceremonies for their reported psychedelic effects, in areas endemic for leishmaniasis diseases. This work extends the studies of Evans and Croft (1987) who evaluated antileishmanial activity, using L. mexicana, of harmaline and other tryptamine derivatives [22]. In vitro, harmaline was the most potent compound tested with an $\mathrm{ED}_{50}$ of $99 \mu \mathrm{M}$. They speculated that the target enzyme is an enzyme involved in aromatic amino acid catabolism. In our studies, we have selected to extend these early studies but using the promastigote form of the non-human species, L. tarentolae which has been validated as a model system to screen drug candidates with lower costs and more safely [23]. In addition, effects of these compounds to cell free supernatant, containing secreted acid phosphatases (SAPs) can be easily evaluated. Both the cell viability and SAP enzyme measurements can be made at various points in the growth curve to assess if there are culture age dependent effects.

Harmine and harmaline are found in the Peganum harmala plant, also known as Syrian Rue, as well as Banistreriopsis caapi vine, which is the key ingredient in Ayahuasca. Ayahuasca is a drink that is traditionally used for ritual, medical, and recreational uses. The beverage is prepared by boiling the Banistreriopsis stems, and an

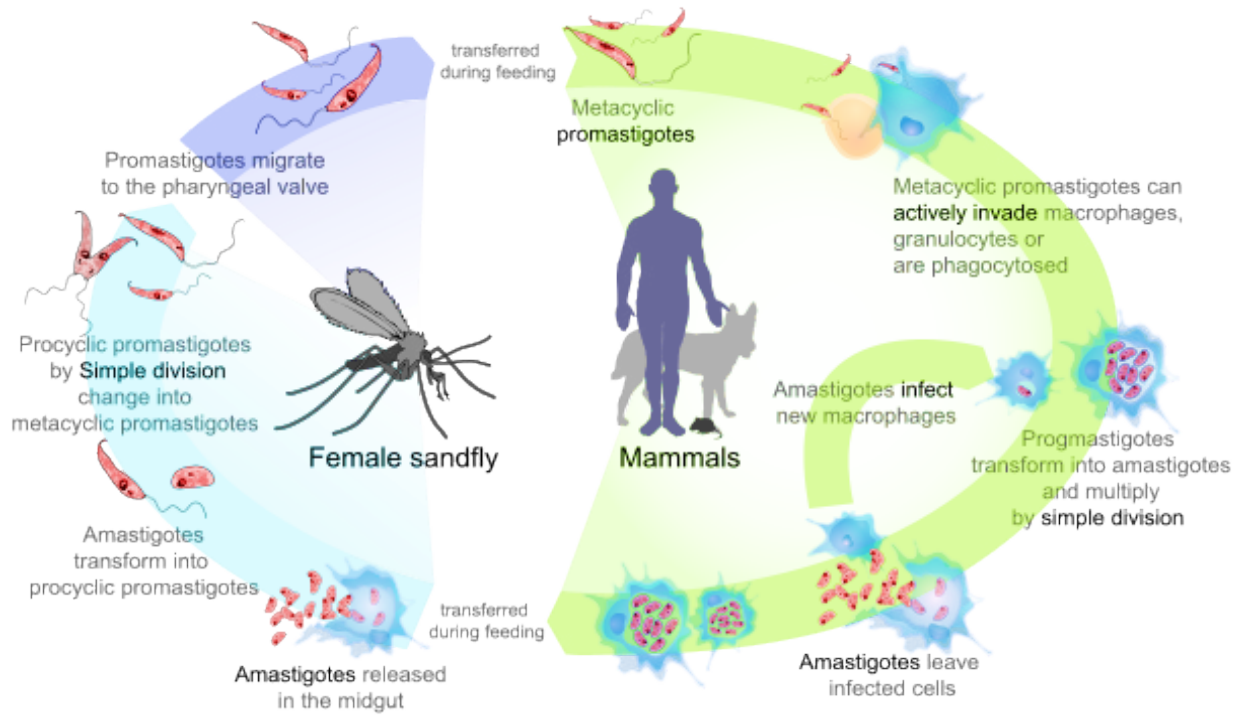

Figure 1. The Leishmania life cycle (modified from www.cdc.gov/parasites/leishmaniasis/index.html [8]) 
2a. Tryptamine<smiles>NCCc1c[nH]c2ccccc12</smiles>

2b. Harmine

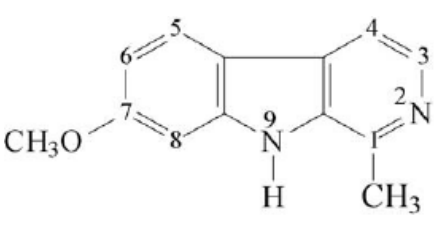

2c. Harmaline<smiles></smiles>

Figure 2. Structures of test compounds

average dose contains about $460 \mathrm{mg}$ of harmine and $41 \mathrm{mg}$ of harmaline [24]. Harmine and harmaline are abundant in the roots and seeds of both Peganum harmala and Banistreriopsis caapi, but the root has the highest concentration of both compounds. Both compounds have a dose dependency degree of toxicity. A case report has shown that a 100$150 \mathrm{~g}$ oral dose of the harmala seeds can result in severe adverse effects, including vomiting of blood and convulsions [24].

\section{Methods}

\section{Culturing of $L$. tarentolae Parasites with and without the Addition of Tryptamine or beta-Carboline Alkaloid Derivatives and Viability Assays}

Axenic Leishmania tarentolae (ATCC 30143) promastigote cells were cultured under sterile conditions in brain heart infusion (BHI; $37.0 \mathrm{~g} / \mathrm{L}$; Sigma Aldrich) medium supplemented with hemin (10 $\mu \mathrm{M}$,$) , penicillin (10,000 \mathrm{U} / \mathrm{mL})$ with streptomycin $(10 \mathrm{mg} / \mathrm{mL}$; Sigma Aldrich) at room temperature $\left(21-23{ }^{\circ} \mathrm{C}\right)$ for three days following the methods of Morganthaler, et al. [25] in $60 \mathrm{~mL}$ stock cultures. On the third day, the cells were divided into $10 \mathrm{~mL}$ aliquots and grown in canted culture flasks $\left(25 \mathrm{~cm}^{2}\right.$, Corning, Inc.) with addition of either DMSO, tryptamine, harmine, or harmaline (purchased from Sigma Aldrich and used as received) at concentrations from 0 to $250 \mu \mathrm{M}$. As shown in the time course of cell growth, the early lag phase is from days $1-3$, the exponential phase is from days 3-6, and the stationary phase involves days 6-7 with some cultures entering senescence phase by day 7. The range of concentrations (especially the lower levels) used were selected relative to the general in vivo estimates of Ayahuasca levels of harmaline measured in volunteers 2 hours after consuming ayahuasca [17]. In all cases, compounds were added on day 3 of culture.

All compounds were made at the stock concentration of $25 \mathrm{mM}$ in dimethylsulfoxide (DMSO). When diluted appropriately, the final concentration of DMSO in cell cultures was always equal to 1 $\%(\mathrm{v} / \mathrm{v})$. These $L$. tarentolae cell cultures with compound additions were allowed to grow at room temperature $\left(21-23^{\circ} \mathrm{C}\right)$ before samples were taken for assessment by the [3-(4,5-dimethylthiazol-2-yl)-2,5diphenyltetrazolium bromide (MTT; Sigma Aldrich) viability assay [26]. For each sample type, a representative cell sample and MTT reagent were incubated for 60 minutes in a flat well 96 well polypropylene plate (Fisher). The absorbance of samples was measured at $595 \mathrm{~nm}$ using an iMark microplate reader (BioRad Laboratories, Hercules, CA). BHI medium alone served as a blank with its value subtracted from sample (BHI + Cells) absorbance values. The sample results are reported as corrected mean \pm standard deviation $(n=3)$. These MTT assays were conducted on days 3-7 of culture and before addition of compounds, all flasks yielded the same MTT value indicating same general cell number and cell viability so that MTT values after addition of compounds can be compared for any change in the MTT value. Also, on culture days
5,6 , and 7, additional aliquots were taken and prepared for assessment for the secreted acid phosphatase enzyme assay using the general procedure of Mendez, et al. [27].

\section{L. tarentolae Secreted Acid Phosphatase (SAP) Enzyme Assay}

Representative samples of each of the L. tarentolae experimental flasks were taken on days 5,6 , and 7 for assessment by the secreted acid phosphatase enzyme assay. Samples $(2 \mathrm{~mL}, \mathrm{n}=3)$ were taken from the corresponding $L$. tarentolae cell culture flask representing each experimental condition, and centrifuged $\left(2000 \mathrm{xg}, 10^{\circ} \mathrm{C}, 8 \mathrm{~min}\right)$. The cell free supernatants were collected and rested on ice until their use in the enzyme assay. SAP assays were performed in $1.5 \mathrm{~mL}$ polypropylene tubes. For days 5 and 6, aliquots $(450 \mu \mathrm{L})$ from each of the harvested enzyme samples were combined with sodium acetate buffer $(450 \mu \mathrm{L}$, $\mathrm{pH} 4.5,0.5 \mathrm{M})$. The assay was initiated by the addition of the artificial substrate para-nitrophenyl phosphate ( $p \mathrm{NPP} ; 100 \mu \mathrm{L}$ of $5 \mathrm{mg} p \mathrm{NPP} / \mathrm{mL}$ buffer, Sigma Aldrich). Assays were incubated at room temperature for 24 hours which has been previously determined as apparent first order conditions [27]. For day 7 , aliquots $(100 \mu \mathrm{L})$ from each of the harvested enzyme pools were combined with sodium acetate buffer $(800 \mu \mathrm{L}, \mathrm{pH}$ $4.5,0.5 \mathrm{M})$. All enzyme reactions were stopped with sodium hydroxide $(100 \mu \mathrm{L}, 10 \mathrm{M})$. The samples were then vortexed and product formation was measured by UV Vis Spectroscopy at $405 \mathrm{~nm}$. In all cases, BHI medium with the appropriate concentration of the corresponding test compound was used as a spectrophotometric blank. Data are reported as percent change relative to same age control cells. Sample replicates were performed as $n=3$.

\section{Microscopy}

On days 3,4, and 5 of culture, cells were assessed for motility, cell shape, and clumping using a Jenco International, Inc. (Portland OR) inverted compound microscope Model CP-2A1 at 100x and $400 \mathrm{x}$. Images were captured daily using a Google Pixeled cellular phone (model G-2PW4100).

\section{Results}

\section{Effect of Tryptamine, Harmine or Harmaline Compounds on L. tarentolae Cell Motility, Cell Shape, and Cell Clumping}

There were no apparent changes in cell motility or shape in treated cells relative to control cells in these trials. However, cells of day 3, 4, and 5 cultures exposed to either harmine, harmaline, or tryptamine exhibit clumping differences relative to control cells. Relative to control cells, exposure to harmine, harmaline, or tryptamine resulted in about a $33 \%, 25 \%$ or $60 \%$ increase, respectively, in number of cell clumps on day 5 (data not shown). This is of interest as the cell clumping is reflective of cell stress [28]. Also, the apparent clump size (reflective of the number of cells per clump) and clump shape were not consistent in the test flasks relative to the control cell flasks. 


\section{Effect of Test Compounds on L. tarentolae Cell Viability}

In the presence of harmine (from 12.50 to $250.0 \mu \mathrm{M}$ final concentration), the cell viability was negatively affected at and above $62.50 \mu \mathrm{M}$ on days 4,5 , and 6 of days in culture. The cultures exposed to $62.50 \mu \mathrm{M}$ did appear to recover on day 7 whereas above that concentration, cell viability remained very low as shown in Figure 3. As shown in Figure 4, the same trend is shown with tryptamine. The cells exposed to $12.50 \mu \mathrm{M}$ did exhibit an apparent recovery effect not observed for any other concentration of tryptamine. For cells exposed to the various concentrations of harmaline, the cell viability, shown in Figure 5, appears less inhibited by addition of this compound relative to the other two test compounds.

\section{Effect of Tryptamine Compounds on L. tarentolae Secreted Acid Phosphatase Activity (SAP)}

The effects of the indole derivatives on the amount of detectable secreted acid phosphatase activity in cell free supernatants are shown in Figures 6-8 for harmine, tryptamine, and harmaline, respectively. In the cases of harmine and tryptamine, low levels of these compounds appeared to result in more detectable SAP activity relative to control cell supernatant enzyme activity whereas the highest compound levels results in less detectable enzyme activity. In contrast, low levels of harmaline resulted in less detectable activity (but at comparable levels found from control cells) than at higher levels of the compound that resulted in substantially higher amounts of detectable enzyme activity especially on day 6 .

\section{Discussion}

\section{Effect of Tryptamine Compounds on L. tarentolae Cell} Viability, Motility, Cell Shape, and Cell Clumping

While addition of these compounds did not appear to affect cell motility or general shape, by Day 5 there was a noticeable effect of cell

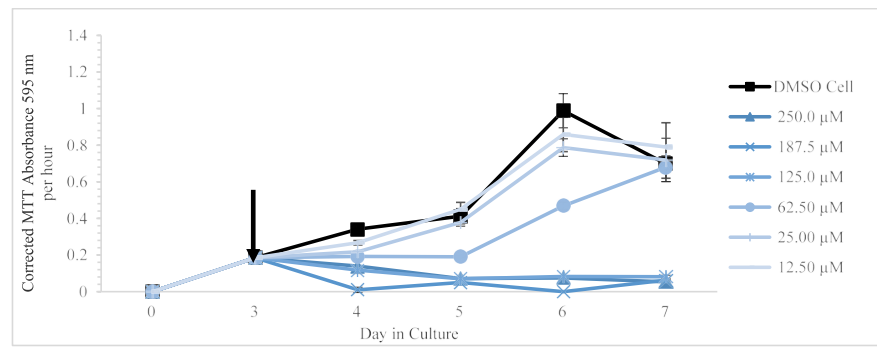

Figure 3. Corrected MTT absorbance values (absorbance at $595 \mathrm{~nm}$ per 1 hour of incubation with the MTT reagent) as a function of days in culture is shown as mean and $\mathrm{SD}$ for $\mathrm{n}=3$ replicates. The arrow indicated the time of addition of DMSO only or harmine dissolved in DMSO

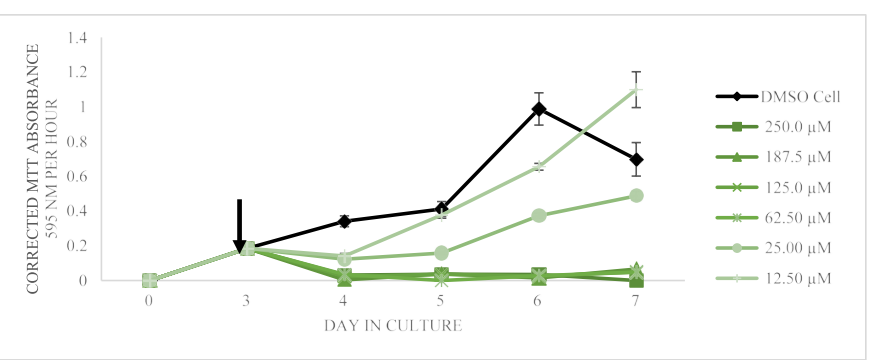

Figure 4. Corrected MTT absorbance values (absorbance at $595 \mathrm{~nm}$ per1 hour of incubation with the MTT reagent) as a function of days in culture is shown as mean and SD for $n=3$ replicates (error bars less than $5 \%$ are not shown). The arrow indicated the time of addition of DMSO only or tryptamine dissolved in DMSO

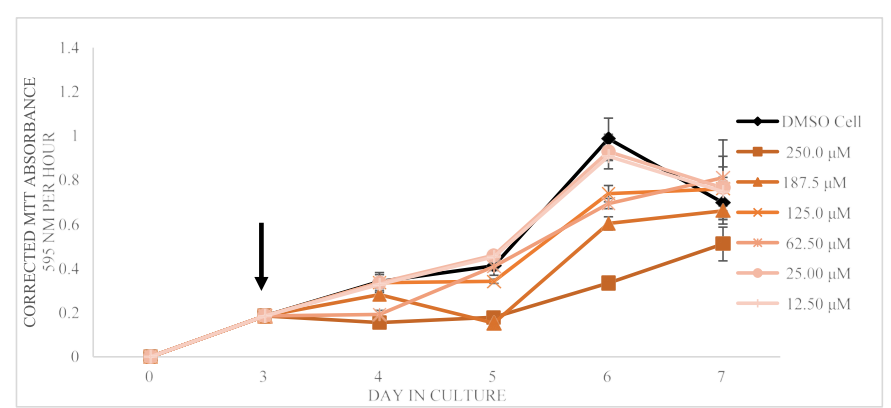

Figure 5. Corrected MTT absorbance values (absorbance at $595 \mathrm{~nm}$ per1 hour of incubation with the MTT reagent) as a function of days in culture is shown as mean and SD for $n=3$ replicates (error bars less than $5 \%$ are not shown). The arrow indicated the time of addition of DMSO only or harmaline dissolved in DMSO

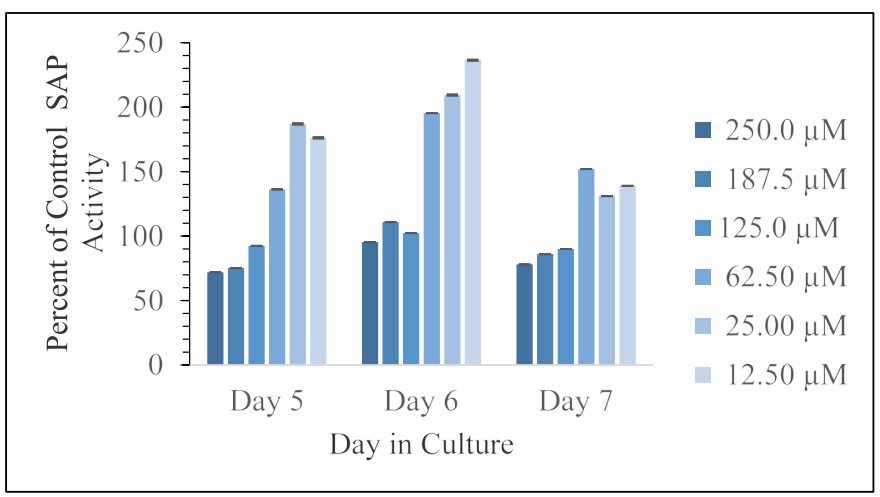

Figure 6. Corrected absorbance values using the supernatant fraction from cells exposed to the various concentrations of harmine as percent of control cells, with DMSO only, as a function of days in culture is shown as mean and SD for $\mathrm{n}=3$ replicates. Error bars are shown and in all cases were less than $5 \%$ of the mean value

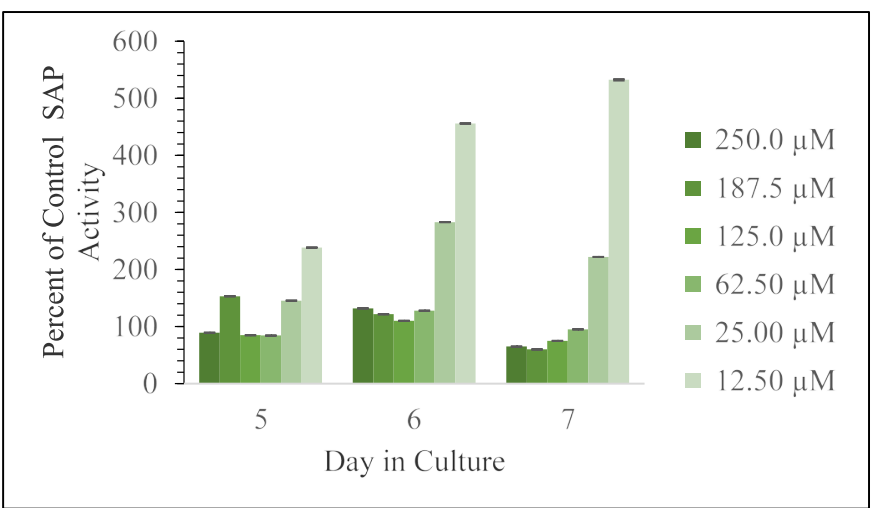

Figure 7. Corrected absorbance values using the supernatant fraction from cells exposed to the various concentrations of tryptamine as percent of control cells, with DMSO only, as a function of days in culture is shown as mean and SD for $n=3$ replicates. Error bars are shown and in all cases were less than $5 \%$ of the mean value

clumping relative to control (DMSO) cells with the most noticeable change being seem with the addition of tryptamine. Relative to control cells, Day 5 cells with tryptamine had some $60 \%$ more clumps. However, the clump size and shape appeared to be varied relative to the control cells. Cells with DMSO addition appeared to reach the stationary phase in the growth curve by day 6 (as indicated by the MTT values), whereas, depending on the concentration and type of compound, this stationary phase was reached at different times and to different extents. The results of the MTT assays clearly indicate that the three test compounds investigated here are active against axenic $L$. tarentolae and cause 


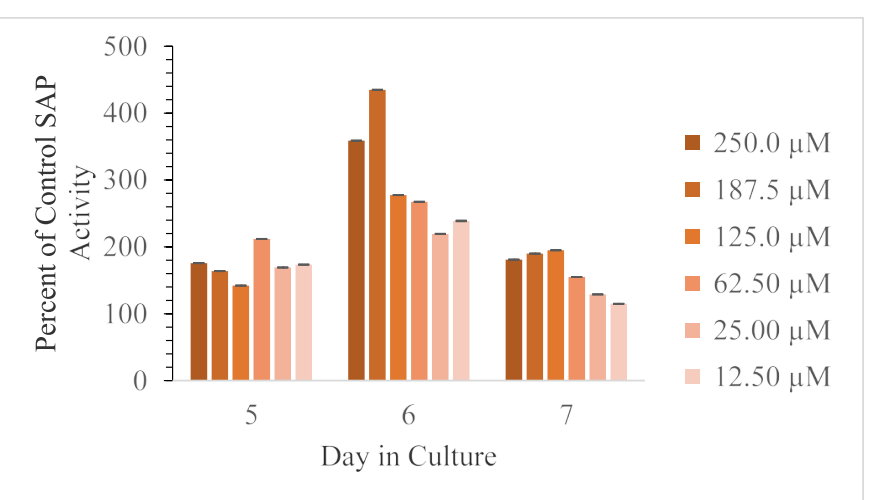

Figure 8. Corrected absorbance values using supernatant fraction cells exposed to the various concentrations of harmaline as percent of control cells, with DMSO only, as a function of days in culture is shown as mean and SD for $\mathrm{n}=3$ replicates. Error bars are shown and in all cases were less than $5 \%$ of the mean value

measurable decreases in cell MTT values in a dose dependent manner. In general, exposure to increasing amounts of any of the three indole compounds led to decreases in L. tarentolae cell MTT values by 24 hours post addition as measured by the MTT viability assay. Harmaline exposure led to the smallest negative effect on cell viability and/or cell number as assessed by the MTT values both in magnitude of any single dose compared to control, and average inhibition.

The largest decrease in MTT values caused by exposure to harmaline $(250.0 \mu \mathrm{M})$ occurred on day 7 of cell culture, and decreased cell viability by $27 \%$ relative to control cells. Thus, exposure to harmaline had a smaller effect on cell viability compared to the largest decreases caused by either tryptamine $(250.0 \mu \mathrm{M}$, day $7,100 \%$ decrease of viability relative to control cells) or harmine ( $250 \mu \mathrm{M}$ day $92 \%$ decrease of viability of control cells). Furthermore, the average inhibition over the entire test period of $L$. tarentolae cell viability caused by each compound, listed from greatest inhibition to least inhibition, was tryptamine (74\% decrease vs. control), harmine ( $52 \%$ decrease vs. control), harmaline ( $17 \%$ decrease vs. control). Thus, our data confirm the report by Evans and Croft [22] that harmaline decreases cell viability. However, the largest decrease (67\%) was on day 6 of culture and the culture appears to recover on day 7 .

Not all compound doses led to decreases in L. tarentolae cell MTT values across the entire cell culturing period. The experimental design was such that the compounds were added to the $L$. tarentolae culturing medium only once. The parasites were then exposed to this medium continuously over the entire duration of their cell culture (4 more days). Doing this led to an apparent recovery effect, in some cases. Even more interesting to note, is that the frequency of the parasite recovery to compound exposure trends inversely with the compound effectiveness as an average inhibitor. The larger the average inhibition on $L$. tarentolae cell viability that a compound had, the less the cell culture appeared to recover (Table 1). Furthermore, the better a compound was at acting as an average inhibitor on L. tarentolae cell viability, the more likely that the recovery effect to that compound was to occur at the lowest micromolar exposure (Table 1) implying that the cells that were resistant to the compound (mechanism unknown) were able to continue growing and dividing. It is interesting to note that the recovery from the most toxic compound in this work, tryptamine, exhibited the largest effect observed. This suggests the compounds have the ability to lead to selection of resistant parasites with frequent low exposures.

One of the more surprising results from this work was the large difference in effect on $L$. tarentolae cell MTT values that was observed after harmine or harmaline exposure. The structures of harmine (Figure $2 \mathrm{~b}$ ) and harmaline (Figure $2 \mathrm{c}$ ) are very similar, but exposure to harmine led to both greater magnitude of effect by any single dose compared to control cells, and greater average inhibition than harmaline. Structurally, both compounds have a double bond between carbon 1 and nitrogen 2 . These compounds differ in that harmine has a double bond between carbon 3 and carbon 4, where harmaline is saturated. This additional carbon-carbon double bond in harmine leads to a larger aromatic system of electrons than is found in the harmaline molecule. This increased stability may prevent or create a reduction in the rate of harmine metabolism and may be a contributing factor to harmine's greater inhibitory effect on cell MTT values. Tryptamine was the best inhibitor of $L$. tarentolae cell MTT values. It had the greatest inhibitory effect at any single time point compared to control, except on day 7 at the lowest concentration tested, and the greatest effect on average inhibition. Furthermore, it was consistently the best inhibitor tested across all dosages and most exposure times. Hence, we speculate that the indole class of molecules may be a rich source of molecular inhibitors of Leishmania cell viability.

\section{Effect of Tryptamine Compounds on L. tarentolae Secreted Acid Phosphatase Activity (SAP)}

The results of the SAP assays testing $L$. tarentolae enzyme pools from days 5,6 , and 7 of cell culture show that these compounds have direct effects on SAP activity. The average effect (data not shown) of these compounds is to increase detectable SAP activity (either as a result of more enzyme secretion from each cell or from having more cells): Harmine ( $131 \%$ of control), Harmaline ( $211 \%$ of control), and Tryptamine (171\% of control).

The minimum and maximum effect of these indole compounds on SAP activity are as follows: harmine (Day 5, $250.0 \mu \mathrm{M}, 72 \%$ of control; Day 6, $12.5 \mu \mathrm{M}, 236 \%$ of control); harmaline (Day 7, $12.5 \mu \mathrm{M}, 115$ $\%$ of control; Day $6,187.5 \mu \mathrm{M}, 435 \%$ of control); tryptamine (Day 7, $187.5 \mu \mathrm{M}, 60 \%$ of control; Day 7, $12.5 \mu \mathrm{M}, 533 \%$ of control). Thus, the greatest inhibition was observed from harmine exposure. The greatest activation was observed from tryptamine exposure.

Generally, with increasing doses of harmine and tryptamine, the detection of SAP activity decreased. Harmine has inhibitory effects on Days 5 and 7 at $125.0 \mu \mathrm{M}, 187.5 \mu \mathrm{M}$, and $250.0 \mu \mathrm{M}$ concentrations. Tryptamine has inhibitory effects on Day $5(62.5 \mu \mathrm{M}, 125.0 \mu \mathrm{M}$, and $250.0 \mu \mathrm{M})$ and Day $7(62.5 \mu \mathrm{M}, 125.0 \mu \mathrm{M}, 187.5 \mu \mathrm{M}$, and 250.0 $\mu \mathrm{M})$. Harmaline exposure led exclusively to activation. Harmaline activation doubles from Day 5 to Day 6, and then decreases by half on Day 7. Interestingly, the compounds that are structurally most similar, harmine and harmaline, do not affect SAP activity to the same degree.

Table 1. The conditions under which $L$. tarentolae cell viability recovered from exposure to compounds

\begin{tabular}{|c|c|c|c|}
\hline Compound & Concentration $(\boldsymbol{\mu M})$ & Day in Culture & $\begin{array}{c}\text { Cell Viability as a } \\
\text { Percent of the Same } \\
\text { Day Control }\end{array}$ \\
\hline \multirow{2}{*}{ Tryptamine } & 12.5 & 7 & 157 \\
\hline \multirow{2}{*}{ Harmine } & 12.5 & 7 & 113 \\
\cline { 2 - 4 } & 25.0 & 7 & 103 \\
\hline \multirow{3}{*}{ Harmaline } & 12.5 & 5 & 110 \\
\cline { 2 - 4 } & 25.0 & 5 & 112 \\
\cline { 2 - 4 } & 12.5 & 7 & 107 \\
\cline { 2 - 4 } & 25.0 & 7 & 110 \\
\cline { 2 - 4 } & 62.5 & 7 & 116 \\
\hline
\end{tabular}


Harmaline exposure led exclusively to activation of SAP activity, but harmine exposure led to activation or inhibition depending on dose.

\section{Conclusions}

The effects of these indole derivatives on Leishmania in the context of leishmaniasis are potentially concerning. The effects on $L$. tarentolae cell viability and SAP activity appear dose dependent. Tryptamine was the most effective compound at inhibiting L. tarentolae cell MTT values, on average, yet at the lowest concentration tested exhibited an apparent recovery effect on culture viability and subsequent growth. Unfortunately, it also appears to be an excellent activator of SAP activity, on average; however, more studies are needed to establish this direct effect. Harmine was the next best inhibitor of $L$. tarentolae cell viability on average. Harmine was also the weakest activator of SAP activity on average. Harmaline was least effective at inhibiting $L$. tarentolae cell viability on average. Harmaline is also the compound that increased SAP activity the most, on average. If the secreted acid phosphatase has an important role in parasite infectivity as reported by others $[10,12]$, increasing the activity of this enzyme may counteract the apparent toxic effect of cell viability that these compounds exhibited. Thus, it may be the case that those infected with leishmaniasis are worsening their condition by exposure to these compounds used in ceremonies. We recommend that more epidemiological studies be done to evaluate these potential effects.

\section{Conflicts of Interest}

The authors declare no conflicts of interest.

\section{Author Contributions}

All authors contributed to experimental design, obtaining data, interpreting data and writing the manuscript.

\section{Acknowledgment}

The authors acknowledge support the Illinois State University Department of Chemistry for this work.

\section{References}

1. https://www.cdc.gov/parasites/leishmaniasis/gen_info/faqs.html

2. https://www.cdc.gov/parasites/leishmaniasis/epi.html

3. Bekhit A, El-Agroudy E, Helmy A, Ibrahim RM, Shavandi A, et al. (2018) Leishmania treatment and prevention: Natural and synthesized drugs. European J Med Chem 160: 229-244.

4. https://www.cdc.gov/parasites/leishmaniasis/disease.html

5. https://www.cdc.gov/parasites/leishmaniasis/health_professionals/index.html

6. Walton BC, Valverde L (1979) Racial differences in espundia. Ann Trop Med Parasitol 73: 23-29. [Crossref]

7. Assis TSM, Rosa DCP, Teixeira EdeM, Cota, Azeredo daS, et al. (2017) The direct costs of treating human visceral leishmaniasis in Brazil. Rev Soc Bras Med Trop p. 4.
8. https://www.cdc.gov/parasites/leishmaniasis/biology.html

9. Mojtahedi Z, Clos J, Kamali-Sarvestani E (2008) Leishmania major: Identification of developmentally regulated proteins in procyclic and metacyclic promastigotes. Exp Parasitol 119: 422-475.

10. Vannier-Stantos MA, Martiny A, Meyer-Fernandes JR, Desouza W (1995) Leishmanial protein kinase $\mathrm{C}$ modules host cell infection via secreted acid phosphatase. Eur J Cell Biol 67: 112-119.

11. Wiese M, Ilg T, Lottspeich F, Overath P (1995) Ser/Thr-rich repetitive motifs as targets for phosphoglycan modifications in Leishmania mexicana secreted acid phosphatase. EMBO J 14: 1067-1074.

12. Soulat D, Bogdan C (1898) Function of macrophage and parasite phosphatases in leishmaniasis. Front Immunol.

13. McCall LI (2012) Parasite and Host Determinants of Visceral Leishmanasis. Thesis, McGill University, Canada.

14. https://pubchem.ncbi.nlm.nih.gov/compound/tryptamine\#section=Top

15. Araújo AM, Carvalho F, Bastos Mde L, Guedes de Pinho P, Carvalho M (2015) The hallucinogenic world of tryptamines: an updated review. Arch Toxicol 89: 1151-1173. [Crossref]

16. Schultes RE (1970) The plant kingdom and hallucinogens (part III). Bull Narc 12: 25 53.

17. Riba J, Valle M, Urbano G, Yritia M, Morte A, et al. (2003) Human pharmacoloy of ayahuasca: subjective and cardiovascular effects, monoamine metabolite excretion, and pharmacokinetics. J Pharm Experi Thera 306: 73-83.

18. Stollak JS, Furchgott RF (1983) Use of selective antagonists for determining the types of receptors mediating the actions of 5-hydroxytryptamine and tryptamine in the isolated rabbit aorta. J Pharmacol Exp Ther 224: 215-221.

19. Terron JA, Falcon N (1999) Pharmacological evidence for the 5-HT7 receptor mediating smooth muscle relaxation in canine cerebral arteries. Br J Pharmacol 127: 609-616.

20. Sanders B, Lankenau SE, Bloom JJ, Hathazi D (2008) Research chemicals: Tryptamine and phenylethylamine use among the high-risk youth. Subst. Misuse 43: 930-937.

21. Shazia K, Anjuma SN, Jaleela F, Khana J, Naseema S (2017) Biomedical significance of tryptamine: A review." J Pharmacovigil 5: 5.

22. Evans AT, Croft SL (1987) Antileishmanial activity of harmaline and other tryptamine derivatives. Phytother Res 1: 25-27.

23. Taylor VM, Muñoz DL, Cedeño DL, Vélez ID, Jones MA, et al. (2010) Leishmania tarentolae: Utility as an in vitro model for screening of antileishmanial agents. Experi Parasitol 126: 471-475.

24. Mahmoudian M, Jalilpour H, Salehian P (2002) Toxicity of Peganum harmala: Review and a case report. Iranian J Pharm \& Therapeu 1: 1-4.

25. Morganthaler JB, Peters SJ, Cedeno DL, Constantino MH, Edwards KA, et al. (2008) Carbaporphyrin ketals as potential agents for a new photodynamic therapy treatment of leishmaniasis. Bioorg Med Chem 16: 7033-7038.

26. Mosmann T (1983) Rapid colorimetric assay for cellular growth and survival: Application to proliferation and cytotoxicity assays. J Immunol Methods 65: 55-63.

27. Mendez RS, Dorsey BM, McLauchlan CC, Beio M, Turner TL, et al. (2014) Vanadium complexes are in vitro inhibitors of Leishmania secreted acid phosphatases. Int J Chem 6: 35-49.

28. Dorsey BM, Cass CL, Cedeno DL, Vallejo R, Jones MA (2018) Effects of specific electric field stimulation on the release and activity of secreted acid phosphatases from Leishmania tarentolae and implications for therapy. Pathogens 7: 77-90.

Copyright: (C2019 Wickline J. This is an open-access article distributed under the terms of the Creative Commons Attribution License, which permits unrestricted use, distribution, and reproduction in any medium, provided the original author and source are credited. 